\title{
The origin of the hot metal-poor gas in NGC $1291^{\star}$
}

\section{Testing the hypothesis of gas dynamics as the cause of the gas heating}

\author{
I. Pérez ${ }^{1}$ and K. Freeman ${ }^{2}$
}

\author{
1 Kapteyn Astronomical Institute, University of Groningen, Postbus 800, Groningen 9700 AV, The Netherlands \\ e-mail: isa@astro.rug.nl \\ 2 RSAA, Mount Stromlo Observatory, Cotter road, Weston Creek, ACT 2611, Australia
}

Received 12 January 2005 / Accepted 11 April 2006

\begin{abstract}
In this paper we test the idea that the low-metallicity hot gas in the centre of NGC 1291 is heated via a dynamical process. In this scenario, the gas from the outer gas-rich ring loses energy through bar-driven shocks and falls to the centre. Heating of the gas to X-ray temperatures comes from the high velocity that it reaches $\left(\approx 700 \mathrm{~km} \mathrm{~s}^{-1}\right)$ as it falls to the bottom of the potential well. This would explain why the stellar metallicity in the bulge region is around solar while the hot gas metallicity is around 0.1 solar. We carried out an observational test to check this hypothesis by measuring the metallicity of HII regions in the outer ring to check whether they matched the hot gas metallicity. For this purpose we obtained medium resolution long slit spectroscopy with FORS1 on the ESO VLT at Paranal and obtained the metallicities using emission line ratio diagnostics. The obtained metallicities are compatible with the bulge stellar metallicities but very different from the hot-gas metallicity. However, when comparing the different time-scales, the gas in the ring had time enough to get enriched through stellar processes, therefore we cannot rule out the dynamical mechanism as the heating process of the gas. However, the blue colours of the outer ring and the dust structures in the bar region could suggest that the origin of the X-ray hot gas is due to the infall of material from further out.
\end{abstract}

Key words. galaxies: ISM - galaxies: structure - galaxies: kinematics and dynamics - Galaxy: abundances - X-rays: galaxies

\section{Introduction and hypothesis}

NGC 1291 is an $\mathrm{S}(\mathrm{R}) \mathrm{SB}(\mathrm{s}) 0 /$ a galaxy widely studied due to its brightness $\left(B_{T}=9.39\right.$, de Vaucouleurs et al. 1991), its large angular size $\left(D_{25}=9.8 \mathrm{arcmin}\right)$, and its distance, between 6.9 and $14 \mathrm{Mpc}$. In the blue, the morphology is characterised by a bright inner lens, a primary bar, a small secondary bar misaligned by $\approx 30^{\circ}$ with the primary bar, and a well defined outer ring. The galaxy was first studied in great detail by de Vaucouleurs (1975), who drew attention to the secondary bar as a new phenomenon in barred galaxies. The large outer ring has a diameter around $8^{\prime}$ $(\approx 16 \mathrm{kpc})$. HI data show that the atomic gas is concentrated in the optical ring, with a pronounced central hole (van Driel et al. 1988). The derived total HI mass is $0.81 \times 10^{9} M_{\odot}$ for an adopted distance of $6.9 \mathrm{Mpc}$. NGC 1291 is then relatively gas rich for an S0/a galaxy.

Recent Chandra data (Hogg et al. 2001; Irwin et al. 2002) show that the hot gas is anti-correlated with the HI: the X-ray emission fills the central hole. There are two components in the X-ray emission with evidence for decreasing "hardness" with radius. The Chandra data allow the separation of the stellar emission from the gas emission, showing that the diffuse gaseous component has a ratio $L_{x} / L_{B}=1.3 \times 10^{29} \mathrm{erg} \mathrm{s}^{-1} L_{B_{\odot}}^{-1}$. This luminosity is low, but similar to the values found for $\mathrm{X}$-ray faint ellipticals and the bulge of M 31 . The derived X-ray mass within a radius of $120^{\prime \prime}$ is $5.8 \times 10^{7} M_{\odot}$ (Irwin et al. 2002) which is lower than the average mass of bright ellipticals. They calculated

$\star$ Based on observations obtained at the European Southern Observatory, Paranal, Chile (ESO program 71.B-0458A). a cooling time of the gas at the centre of the galaxy of $1.2 \times$ $10^{7} \mathrm{yr}$ and less than a Hubble time for the hot gas filling the hole.

The unexpected discovery from the Chandra observations is that the derived metallicity of the X-ray emitting gas near the centre of the galaxy is only $0.13 \pm 0.04 \times$ solar. In contrast, an optical measurement of the metallicity of the stellar bulge found it to have a $\mathrm{Mg}_{2}$ index of 0.24 , corresponding to $\mathrm{Fe} / \mathrm{H}=$ $1.1 \times$ solar (Terlevich et al. 1981). Such low X-ray gas abundances have been observed for other early-type galaxies (Sarazin et al. 2001; O'Sullivan \& Ponman 2004). Enrichment of the hot gas in early-type galaxies is generally thought to be due to stellar processes such as type Ia supernovae or stellar mass losses. However, the low metallicity of the hot gas is hard to reconcile with this scenario. Therefore, a new source of heating must be found. Dynamical heating is a possibility. For example, we know that most of the $\mathrm{HI}$ is now in the outer ring. The $\mathrm{HI}$ ring coincides with the stellar ring, and the sharp edges of the ring suggest a dynamical origin, such as a resonance. The bar will have a strong dynamical influence out to the outer Lindblad resonance (OLR, i.e. out to the vicinity of the outer ring of NGC 1291). If one allows for the gas outside the bar to move in non-circular orbits due to the effect of the bar, then the gas can lose angular momentum and can dissipate large amounts of energy through shocks and will fall in toward corotation. If one models the potential field as an isothermal King model, and knowing that the stellar velocity dispersion of the galaxy is about $160 \mathrm{~km} \mathrm{~s}^{-1}$ (Dalle-Ore et al. 1991), one can estimate the infall velocity. For an isothermal, truncated distribution the central potential is $\Phi \approx 9 \sigma^{2}$, 

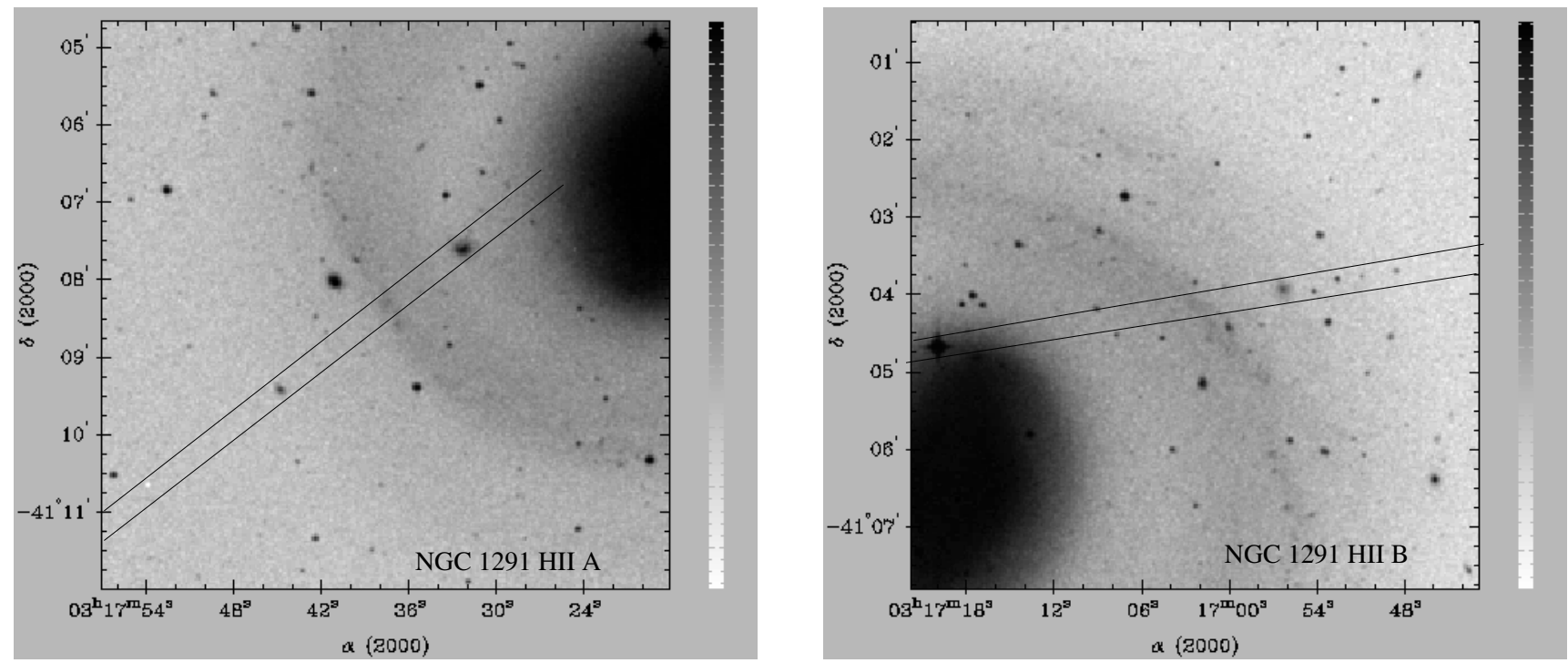

Fig. 1. Long-slit FORS1 observations. The left panel presents the slit position for region A on the south-east side of NGC 1291. The right panel presents the slit position for region B on the north-west side of NGC 1291.

the velocity is then $V=(2|\Phi|)^{1 / 2} \approx 700 \mathrm{~km} \mathrm{~s}^{-1}$. This velocity is enough to heat up the gas to X-ray temperatures as it falls to the bottom of the galaxy's potential well.

In this scenario, the hot metal-poor X-ray gas near the centre of NGC 1291 comes from the gas which we already observed to lie in the outer ring. To test this scenario, we need to find out if the metallicity of the gas in the ring is low and therefore compatible with the metallicity of the X-ray gas near the centre. It is worth noticing that due to the limited survey of X-ray emission in S0/a ringed galaxies, little is known about whether this low metallicity in the hot gas component is a common feature in this type of galaxies.

In this paper we present the observations, data reduction (Sect. 2) and abundance determination (Sect. 3) of a sample of HII regions in the outer ring of NGC 1291. In Sect. 4 we present the results from the abundance determination. The different time-scales at which the different processes taking place occur are presented in Sect. 4.3. Finally, discussion and conclusions are presented in Sect. 5.

\section{Observations and data reduction}

Service observations were carried out at the VLT with FORS1 at the UT1 in August 2003. Two hours were allocated for the project to obtain long slit spectra at two different slit positions on the galaxy with two different instrument configurations (grism 600V and grism 300V). The position of the HII regions were chosen by visual inspection of the DSS image. As already noted by Crocker et al. (1996), only faint HII regions are found in the outer ring in contrast to the inner region where strong $\mathrm{H} \alpha$ emission is found. An image of the two slit positions is shown in Fig. 1. Observations of $1000 \mathrm{~s}$ per slit position with the grism $600 \mathrm{~V}$ were obtained. The wavelength range covered was 4650-7100 $\AA$ with a dispersion of $49 \AA \mathrm{mm}^{-1}$. A second set of exposures of $400 \mathrm{~s}$ were obtained per slit position with the lower resolution $300 \mathrm{~V}$ grism with a wavelength range from $3300-6500 \AA$ and a dispersion of $110 \AA \mathrm{mm}^{-1}$. Standard calibrations were obtained together with three spectrophotometric standards.

Standard data reduction was performed using IRAF routines. All the spectra were bias-subtracted and flat-field corrected with the standard calibration frames provided with the service observations. The spectra were then wavelength calibrated and geometric corrections were applied to correct for any misalignment. The slits were centered on the outer ring so sky subtraction could be carried out using the area outside the galaxy. The spectra were "flattened" using the spectrophotometric standards acquired during the observing run. The fluxes were obtained by fitting a Gaussian to the different emission lines using different spatial binning (see Fig. 2).

\section{Abundance determination}

A few emission lines ([OIII] $5007 \AA$, H $\alpha$, [NII] $6583 \AA$, [SII] 6716 and $6731 \AA$ ) were detected but only in slit position A: see Fig. 1. Since $\mathrm{H} \beta$ and [OII] were not detected, the Balmer decrement cannot be used to estimate extinction, and we cannot use metallicity indicators such as the $[\mathrm{NII}] /[\mathrm{OII}]$ ratio which is among the best diagnostics for high metallicities (Kewley \& Dopita 2002). Nor can we use the ionisation parameter $(q)$ diagnostic ratio $[\mathrm{OIII}] /[\mathrm{OII}]$. We opted for using a combination of the $[\mathrm{NII}] / \mathrm{H} \alpha$ and the $[\mathrm{NII}] /[\mathrm{SII}]$ ratios as presented in Kewley \& Dopita (2002). We made use of the diagrams presented in their paper to locate the data for our HII regions on the theoretical line-ratio vs. metallicity diagrams. The advantage of using these ratios is that the lines are close together, so no dust extinction correction is needed. Furthermore, the $[\mathrm{NII}] /[\mathrm{SII}]$ ratio is unaffected by the absorption lines of the underlying stellar population. As one can see from Fig. 3, at low metallicities the ratio is quite insensitive to metallicity changes. However, it seems a good indicator to discern whether we are in the presence of a high or a low abundance region. This ratio is also dependent on the ionisation parameter, but one can get an estimate of $q$ using the other line ratio, $[\mathrm{NII}] / \mathrm{H} \alpha$. The $[\mathrm{NII}] / \mathrm{H} \alpha$ ratio is very sensitive to shock excitation or the presence of a hard ionising field (i.e. AGN). Also, the $\mathrm{H} \alpha$ emission line will be affected by stellar absorption although, in these regions, we expect it to be an almost negligible effect. In Figs. 3 and 4, the grid computed by Kewley, available on-line from her web page, are shown together with the observed line ratios. The grids correspond to HII region models with ionising radiation derived from stellar atmospheres and stellar evolution codes, non-LTE radiative transfer, 


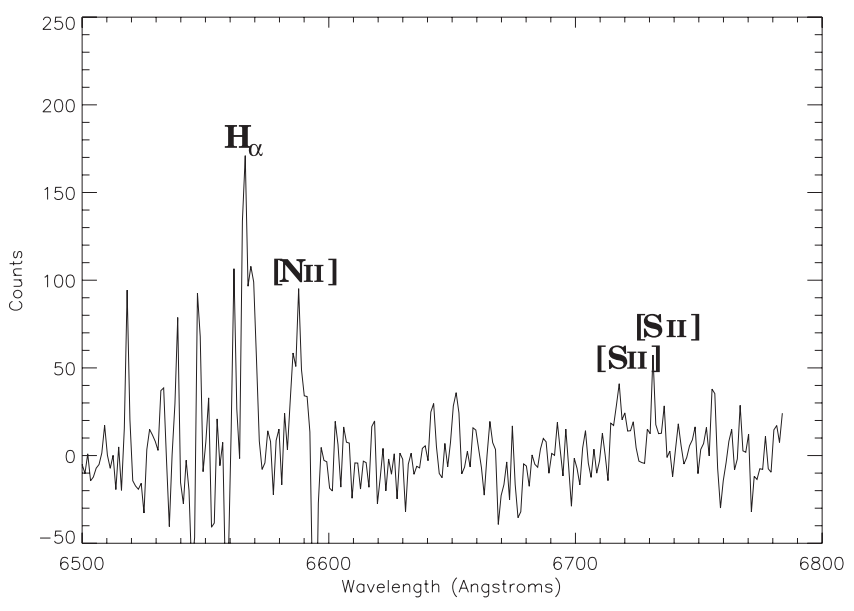

Fig. 2. Spectrum obtained for the HII region situated in the ring region (slit position A), showing the emission lines used in the abundance calculations.

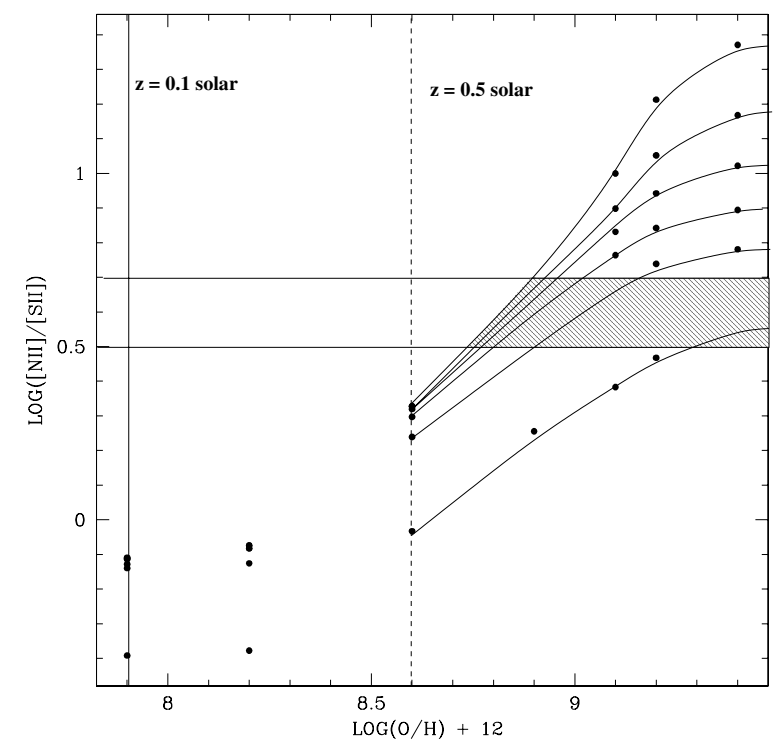

Fig. 3. $\log ([\mathrm{NII}] /[\mathrm{SII}])$ vs. metallicity. Curves are shown for ionisation parameters with $q=\left[5.6 \times 10^{6}, 1.0 \times 10^{7}, 2.0 \times 10^{7}, 4.0 \times 10^{7}, 8.0 \times\right.$ $\left.10^{7}\right]$ from top to bottom, and points with metallicities $0.1,0.2,0.5,1.0$, 1.5, 2.0, 3.0 $Z_{\odot}$ from left to right. The shaded region shows the observed ratios for the ring HII region with error bars included.

and Salpeter IMF, including also a consistent treatment of the dust physics (Dopita et al. 2000). Here, we use the photoionisation grids with the same parameters as in Kewley et al. (2002): $q=\left[5.6 \times 10^{6}, 1.0 \times 10^{7}, 2.0 \times 10^{7}, 4.0 \times 10^{7}, 8.0 \times 10^{7}\right]$ and $Z=0.5,1.0,1.5,2.0$ and $3.0 Z_{\odot}$.

\section{Discussion}

\subsection{On the $X$-ray metallicities}

The X-ray abundances obtained by Irwin et al. (2002) are based on the two-component model consisting of a MEKAL component to represent the gaseous emission and a power law or thermal bremsstrahlung to represent the unresolved stellar sources. The hard-component was fixed at the value found for the sum of the resolved sources. The temperature and metallicity of the MEKAL component were allowed to vary. It has been long argued that the low metallicities found in the X-ray gas of galaxies

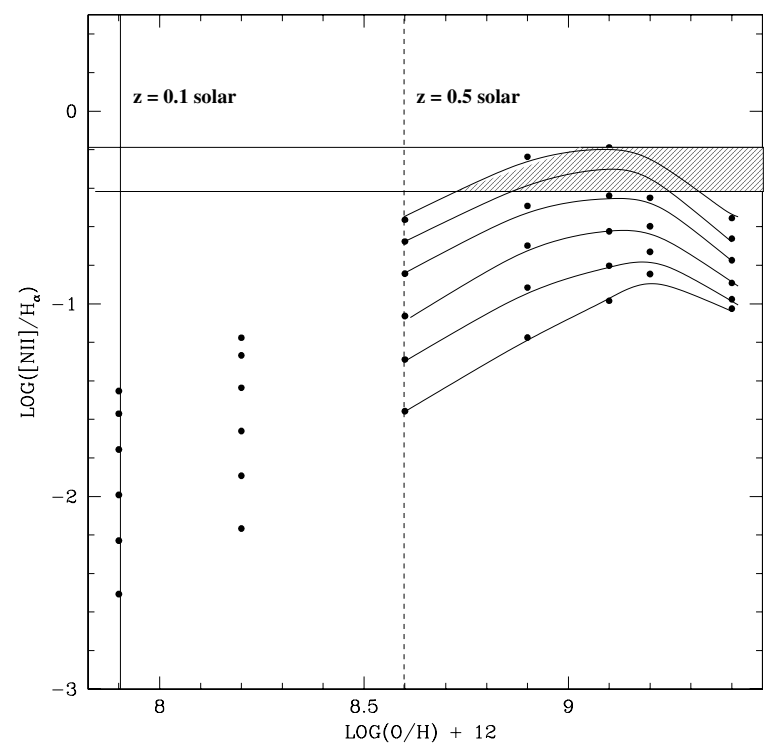

Fig. 4. $\log \left([\mathrm{NII}] / \mathrm{H}_{\alpha}\right)$ vs. metallicity. Curves are shown for ionisation parameter with $q=\left[5.6 \times 10^{6}, 1.0 \times 10^{7}, 2.0 \times 10^{7}, 4.0 \times 10^{7}, 8.0 \times\right.$ $\left.10^{7}\right]$ from top to bottom, and points with metallicities $0.1,0.2,0.5,1.0$, 1.5, 2.0, 3.0 $Z_{\odot}$ from left to right. The shaded region shows the observed ratios for the ring HII region with error bars included. Note that both diagrams give a metallicity for the ring HII region between solar and twice solar, very different to the 0.1 solar metallicity obtained by Irwin et al. (2002) for the hot gas.

are due to the single-temperature fit to the spectra and that a multi-phase fit with higher abundance represents the observed spectra (Buote 2000) equally well. However, this would account for a difference of $\leq 20 \%$ in the metallicity values, which in the case of NGC 1291 cannot explain the low metallicities found.

\subsection{Ionised gas abundances. The results}

Two HII regions were detected in one of the slit positions (position A) while no emission lines were detected along the other slit position (position B), see Fig. 1. On position A, one of the HII regions lies just outside the bulge region, near the end of the bar (hereafter, the "bar region") and the second one lies on the outer ring (hereafter, the "ring region").

Both regions show a high $[\mathrm{NII}] / \mathrm{H}_{\alpha}$ ratio indicating already a metallicity around solar. This result also indicates that the ionisation parameter is low, in the range $1 \times 10^{7}-2 \times 10^{7} \mathrm{~cm} \mathrm{~s}^{-1}$.

The bar region shows [OIII] emission, but no [SII] emission is observed. The $\log ([\mathrm{NII}] /[\mathrm{OIII}])$ ratio is around -0.5 which is normal for HII regions, perhaps a little low, which is to be expected because of the lack of reddening correction, so the combination of the ionisation parameters of $1 \times 10^{7}-2 \times 10^{7} \mathrm{~cm} \mathrm{~s}^{-1}$ and an upper limit $\log ([\mathrm{OIII}] /[\mathrm{NII}])<0.5$ is consistent with a metallicity of solar but not consistent with a metallicity $2 \times$ solar, using the solar abundance value from Anders \& Grevesse (1989). Furthermore, the lack of [SII] emission indicates a high value for the $([\mathrm{NII}] /[\mathrm{SII}])$ ratio which is incompatible with a low metallicity value.

[SII] emission was detected in the inner region giving a $\log ([\mathrm{NII}] /[\mathrm{SII}])$ ratio of about $0.6 \pm 0.1$, again compatible with a metallicity around solar. A value for the ionisation parameters of $1 \times 10^{7}-2 \times 10^{7} \mathrm{~cm} \mathrm{~s}^{-1}$ gives a metallicity incompatible with values higher than $1.2 \times$ solar.

From the results presented in Figs. 3 and 4, the observed abundance of the HII regions is clearly higher than $0.1 \times$ solar. 
The difference between $1 \times$ solar and $0.1 \times$ solar corresponds to a factor of $10 \mathrm{in}[\mathrm{NII}] / \mathrm{H}_{\alpha}$.

\subsection{Time scales}

We find that the metallicity of the ionised regions in the ring of NGC 1291 is around solar. One could argue that the gas in the ring has been enriched after a few episodes of star formation from an initial metallicity close to what is found for the X-ray emitting gas. However, the time-scales of both processes may differ greatly.

To estimate the time scales for the gas to get from the outer disk to the centre we ran a number of SPH simulations in rigid rotating barred stellar potentials (Pérez et al. 2004). The initial particle radial distribution for the gas is a Beta function with a standard deviation set to the scale-length of the disk and radially vanishing at a distance 4 times the scale-length. The non-axisymmetric part of the potential is linearly grown during three bar rotations, so the gas flow can steadily adjust to the forcing of the bar. We observed that, within two bar rotations after the non-axisymmetric component is switched on (one bar rotation before the bar is fully grown), most of the particles are redistributed around angular momenta corresponding to the OLR and the region inside corotation (see Fig. 5). This reflects the dissipation due to the shocks induced by the forcing of the bar. After the non-axisymmetric component is fully grown the gas reaches a stable configuration. Once the gas has settled, the angular momentum distribution remains almost constant. We therefore estimate the outer disk gas infall time-scale to be of the order of two bar rotations.

To estimate the bar rotation speed, we assume that the corotation radius is located at the end of the bar. The observed rotational velocity is uncertain because the galaxy is so nearly faceon (from isophotal analysis we derived an inclination of $6^{\circ} \pm$ $2^{\circ}$ ). From the Tully-Fisher (T-F) relation (Sakai et al. 2000), we can use the apparent magnitudes in $H$ (2MASS) and $B$ (RC3) to estimate the rotational velocity. We derive a rotational velocity at the ends of the bar of $200 \pm 52 \mathrm{~km} \mathrm{~s}^{-1}$ for an adopted distance of $6.9 \mathrm{Mpc}$ (the error does not include the error in the distance). The timescale for two bar rotations is then about $300 \mathrm{Myr}$.

The star formation rates estimated from the $\mathrm{H}_{\alpha}$ fluxes from the discrete HII regions are of the order of $0.1 M_{\odot} \mathrm{yr}^{-1}$ (Caldwell et al. 1991). Using a simple closed-box model the time-scale for the abundance enrichment of the ISM in the ring from $Z \approx 0.1 Z_{\odot}$ to solar metallicities is longer than $1 \mathrm{Gyr}$.

The total optical and near-IR colours (van Driel et al. 1988, 2MASS) of NGC 1291 are typical of early-type galaxies. However, peculiar dust morphology is seen in the lens and the bar region. In the same region, wispy filaments are seen in the ionised gas (Crocker et al. 1996). The outer ring shows bluer colours and also signs of spirality. The gas compression in spiral shocks gives rise to star formation, which would explain the blue colours of the outer ring. The gas content, blue colors and spiral structure of the outer ring might point toward a minor merger event or gas accretion, and therefore an external origin of the hot gas. We note that the cooling time ( Hubble time) is much longer than the enrichment time ( $>1 \mathrm{Gyr})$ and the gas infall time ( $300 \mathrm{Myr})$. It would also be consistent to argue that the hot gas came from the ring at an early time when the ring abundance was low, with the ring becoming enriched through its own subsequent star formation. However, it also remains possible that the metal-poor hot gas comes from the cold reservoir in the outer disk. Unfortunately, there are no bright quasars in the vicinity
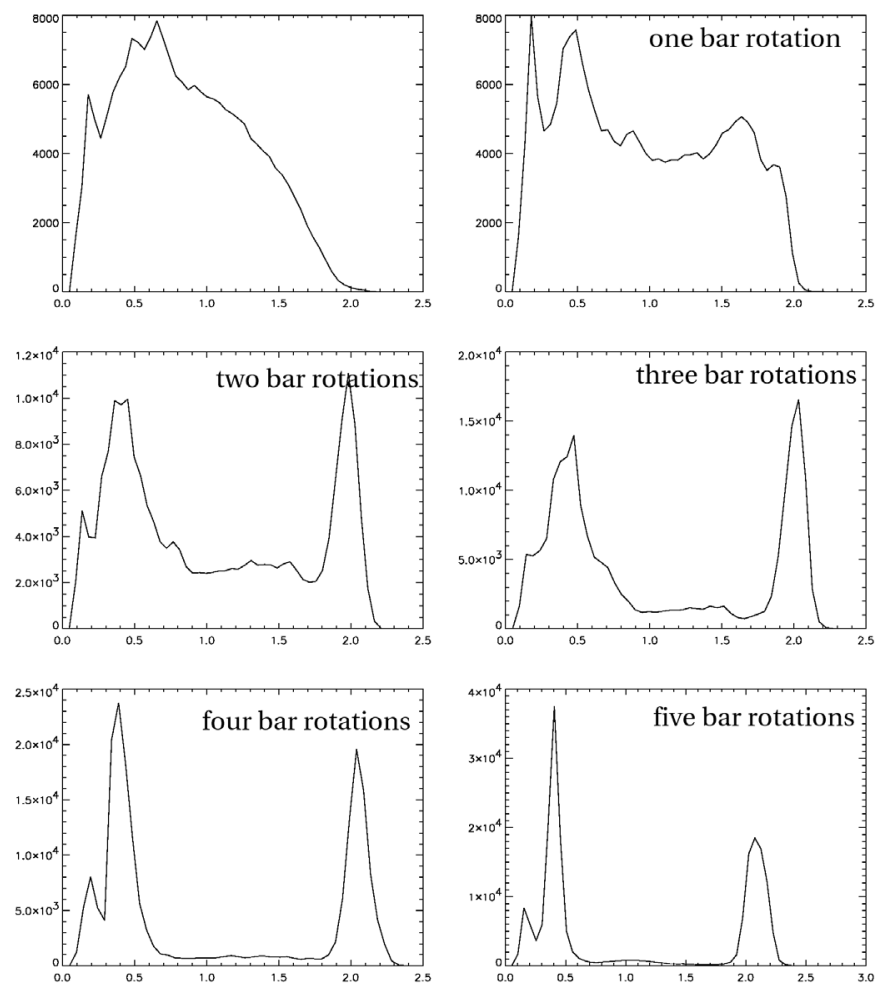

Fig. 5. Time evolution of the number of particles vs. angular momentum distribution in a barred potential. The panels show the distribution up to 5 bar rotations, for a simulation with corotation at the end of the bar. The two peaks correspond to the inner and outer ring respectively.

of NGC 1291 to enable measurement of the abundance of the outermost gas.

\section{Conclusions}

The hot gas enrichment in ellipticals and early-type galaxies is believed to be due mainly to type Ia SNe and stellar mass loss. NGC 1291 has hot gas of low-metallicity at its centre, while having a metal-rich stellar bulge. It is hard to reconcile the low metallicity of the hot gas $(\sim 0.13 \times$ solar $)$ with heating due to stellar processes when the stars have metallicity $\sim 1.1 \times$ solar. We have investigated the possibility that the hot gas comes from the outer ring and is dynamically heated, by measuring the metallicities of two HII regions, one in the ring and one closer to the centre. Both regions show metallicities near solar, much higher than in the the hot gas. Even allowing for a $\approx 20 \%$ error in the X-ray metallicity estimate due to the model dependence of the X-ray abundances, the two metallicities cannot be brought together.

However, when comparing the various timescales, there is time enough for the gas in an initially metal-poor outer ring to become enriched through the usual stellar evolution processes. Therefore, we cannot exclude the possibility that the hot gas comes from an early metal-poor outer ring and is dynamically heated. If the X-ray gas does not come from the ring, as the abundances would at first sight suggest, then it may still come from further out. Further evidence for an external origin would be the presence of peculiar dust structure and ionised gas in the lens and bar region. This infall from external gas could be a common phenomenon, which we happen to detect here in NGC 1291 only because its potential well is so deep. Following the referee's comments and the results from this work, we have started a campaign to do a systematic study on the relation between external 
gas accretion and the metallicity of the X-ray emission in early type galaxies.

Acknowledgements. We thank Lisa Kewley for useful comments and for facilitating the use of her models which have made this work possible. We also thank the service observers at Paranal for the careful observations, and Edwin Valentijn and Michiel Tempelaar who facilitated the use of the ESO-LV catalogue. We further thank the anonymous referee for the useful comments that helped us to improve this manuscript. This research has made use of the NASA/ IPAC Infrared Science Archive, which is operated by the Jet Propulsion Laboratory, California Institute of Technology, under contract with the National Aeronautics and Space Administration. This work has also made use of the 2MASS Atlas Images.

\section{References}

Anders, E., \& Grevesse, N. 1989, Geochim. Cosmochim. Acta, 53, 53, 197 Buote, D. 2000, MNRAS, 311, 176
Caldwell, N., Kennicutt, R., Phillips, A., \& Schommer, R. 1991, ApJ, 370, 526 Crocker, D., Baugus, P., \& Buta, R. 1996, ApJ, 105, 353

Dalle-Ore, C., Faber, S., González, J., Stoughton, R., \& Burstein, D. 1991, ApJ, 366, 38

de Vaucouleurs, G. 1975, ApJS, 49, 193

de Vaucouleurs, G., de Vaucouleurs, A., Corwin, H., et al. 1991, Third Reference Catalogue of Bright Galaxies

Dopita, M., Kewley, L., Heisler, C., \& Sutherland, R. 2000, ApJ, 542, 224

Hogg, D., Morton, R., Bregman, J., \& Haynes, M. 2001, AJ, 121, 1336

Irwin, J., Sarazin, C., \& Bregman, J. 2002, ApJ, 570, 152

Kewley, L., \& Dopita, M. 2002, ApJ, 142, 35

O'Sullivan, E., \& Ponman, T. 2004, MNRAS, 349, 5350

Pérez, I., Fux, R., \& Freeman, K. 2004, A\&A, 424, 799

Sakai, S., Mould, J., Hughes, S., et al. 2000, ApJ, 529, 698

Sarazin, C., Irwin, J., \& Bregman, J. 2001, ApJ, 556, 533

Terlevich, R., Davies, R., Faber, S., \& Burstein, D. 1981, MNRAS, 196, 381

van Driel, W., Rots, A., \& van Woerden, H. 1988, A\&A, 204, 39 